\title{
UNIQUE MINIMALITY OF FOURIER PROJECTIONS ${ }^{1}$
}

\author{
BY
}

\author{
S. D. FISHER, P. D. MORRIS AND D. E. WULBERT
}

\begin{abstract}
The question of when the Fourier projection is the only one of least norm from a space of continuous functions on the circle onto spaces spanned by trigonometric polynomials is studied in two settings. In the first the domain space is the disc algebra and the range is finite-dimensional. In the second the domain space consists of all real continuous functions and the range has finite codimension.
\end{abstract}

\section{Introduction.}

General background. Let $\mathscr{F}_{n}$ be the Fourier projection of the continuous (real or complex) periodic functions, $C_{2 \pi}$, onto $\pi_{n}$ the trigonometric polynomials of degree less than or equal to $n$. One of the extremal properties of $\mathscr{F}_{n}$ is that it has the minimal operator norm along all the projections (i.e. idempotent operators) of $C_{2 \pi}$ onto $\pi_{n}$ (Lozinski [8]). The numbers $\left\|\mathscr{F}_{n}\right\|$ are known to be unbounded. A consequence of this, for example, is that for any sequence of projections $\left\{L_{n}\right\}$ onto $\pi_{n}$-such as interpolation at $n+1$ points-there is an $f$ in $C_{2 \pi}$ and a point $x$ such that $\left(L_{n} f\right)(x)$ is unbounded (Cheney [2]).

The minimal norm property of $\mathscr{F}_{n}$ follows from an averaging process due to Berman [1] which shows that for any projection $P$,

$$
\mathscr{F}_{n} f=\frac{1}{2 \pi} \int_{-\pi}^{\pi} T_{-\lambda} \circ P \circ T_{\lambda} f d \lambda,
$$

where $T_{\lambda} f(x)=f(x+\lambda)$. This equation is also attributed to Marcinkiewicz [9].

The Berman formula immediately shows more than we stated. Let $\Gamma$ be any set of integers and $\pi_{\Gamma}$ the closed linear span of $\left\{e^{i k x}: k \in \Gamma\right\}$. Let $E$ be a subspace of $C_{2 \pi}$ which contains $\pi_{\Gamma}$ and which is the closed linear span of those functions $e^{i n x}$ contained in it. The Fourier projection is then the (obviously unique if it exists) bounded projection $\mathscr{F}_{\Gamma}$ of $E$ onto $\pi_{\Gamma}$ such that

$$
\left(\mathcal{F}_{\Gamma} p\right)(t)=\sum_{j \in \Gamma} \hat{p}(j) e^{i j t}
$$

for all trigonometric polynomials $p$ in $E$. In this setting Berman's formula obtains (since its right-hand side satisfies the defining properties for $\mathscr{F}_{\Gamma}$ ) and so if $P$ is any projection of $E$ onto $\pi_{\Gamma}$ then $\left\|\mathscr{F}_{\Gamma}\right\| \leqslant\|P\|$.

More recently it was found that, for the classical case, $\mathscr{F}_{n}$ is in fact the only projection of minimal norm [3]. It is the extension of this uniqueness property to

Received by the editors February 20, 1980.

1980 Mathematics Subject Classification. Primary 46E15, 42 A05.

Key words and phrases. Fourier projections, continuous function spaces.

${ }^{1}$ Research supported in part by NSF grants. 
other basic settings that is our interest in this study. It is not always true that $\mathscr{F}_{\Gamma}$ is the unique minimal norm projection [6]. Specifically if $\Gamma=\{0, \pm 3, \pm 6, \pm 9, \pm 12\}$ and $P f=\mathscr{F}_{\Gamma}([1+\cos x] f(x))$ then $\|P\|=\left\|\mathscr{F}_{\Gamma}\right\|$. For that matter it is not always true that there are any bounded projections of $C_{2 \pi}$ onto $\pi_{\Gamma}$. From Berman's formula, the Riesz representation theorem, and P. J. Cohen's theorem on idempotent measures (actually Helson's earlier result for our special case) it follows that $\pi_{\Gamma}$ is complemented if and only if $\Gamma$ is in the Boolean algebra generated by groups of integers and their cosets.

Summary of results. This study separates into two distinct settings. One considers projections of the complex analytic function on the disc onto a subspace $\pi_{\Gamma}$ where $\Gamma$ is a finite set of positive integers. It is shown that the Fourier projection is the unique minimal norm projection onto the span of $\left\{e^{i k x}: 0<k<n\right\}$.

The other setting involves only real functions, and has a complete characterization for uniqueness of minimal norm projections onto translation invariant subspaces of finite codimension. That is, in this setting, $\Gamma$ contains all but a finite number of integers and their negatives. For example, similar to the classical settings, uniqueness is obtained when $Z-\Gamma=\{0, \pm 1, \pm 2, \ldots, \pm n\}$. But contrary to the finite analog we also have uniqueness when $Z-\Gamma=$ $\{0, \pm 3, \ldots, \pm 3 n\}$. The minimal norm projection is not unique, for example, when $\mathbf{Z}-\Gamma=\{0, \pm 2, \pm 3, \pm 4\}$. This seems to be the first counterexample that is not of the type described in the beginning paragraphs above.

\section{Part I. Minimal projections of analytic functions}

Let $\Gamma$ be a finite set of nonnegative integers and let $\pi_{\Gamma}$ be the linear span of $\left\{e^{i j x}\right.$ : $j \in \Gamma\}$. Let $A$ be the space of functions $f$ which are continuous on the closed unit $\operatorname{disc} \Delta=\{z:|z| \leqslant 1\}$ and analytic on the interior of $\Delta$. The restriction of $A$ to the unit circle $T$ is a closed subspace of $C(T)$ which we again denote by $A ; A$ is the uniform closure of $\left\{e^{i k x}: k \geqslant 0\right\}$. The Fourier projection $\mathscr{F}_{\Gamma}$ maps $A$ onto $\pi_{\Gamma}$ by the rule

$$
\left(\mathscr{F}_{\Gamma} f\right)\left(e^{i x}\right)=\sum_{j \in \Gamma} \hat{f}(j) e^{i j x}
$$

where

$$
\hat{f}(j)=\frac{1}{2 \pi} \int_{0}^{2 \pi} f\left(e^{i x}\right) e^{-i j x} d x=f^{(j)}(0) / j !, \quad j=0,1, \ldots
$$

We shall show in this part of the paper that when $\Gamma=\{0, \ldots, n\}, \mathscr{F}_{\Gamma}$ is the unique minimal norm projection of $A$ onto $\pi_{\Gamma}$. Unless otherwise indicated all sums in this part of the paper are over the set $\Gamma . \S 1$ of this part closely follows the early development in [3].

1. Necessary conditions for minimality. In this section we make no special assumptions about $\Gamma$. Let $P$ be a projection from $A$ onto $\pi_{\Gamma}$ with minimal norm. Since $P$ is a projection it has the representation

$$
(P f)\left(e^{i x}\right)=\sum e^{i j x} \int_{T} f\left(e^{i t}\right) d \mu_{j}(t)
$$


where $\mu_{j}$ is a finite regular Borel measure on the unit circle $T$. We note that $\mu_{j}$ is unique up to adding an element of $H_{0}^{1}$, the annihilator of $A$ in the space of measures; see [4] for a discussion of this and related facts. Now write $d \mu_{j}=g_{j} d t+$ $d \rho_{j}$ where $g_{j} \in L^{1}$ and $\rho_{j}$ is singular with respect to Lebesgue measure. Berman's equation is

$$
\mathscr{F}_{\Gamma}=\frac{1}{2 \pi} \int_{T} T_{-s} P T_{s} d s,
$$

where $T_{s}$ is the rotation operator: $\left(T_{s} f\right)\left(e^{i x}\right)=f\left(e^{i(x+s)}\right)$. Let $F$ be a function in $H^{\infty}$ which solves the extremal problem

$$
\alpha=\max \left\{\left|\sum f^{(j)}(0) / j !\right|: f \in H^{\infty},\|f\|_{\infty}<1\right\} .
$$

$F$ is unique if we specify that $\sum F^{(j)}(0) / j$ ! be positive. It is known that $F$ is a finite Blaschke product with $n$ or fewer zeros in $\{|z|<1\}$; see $[4,8.4]$. Then $F$ (or a rotation of $F$ ) is the extremal function for $\mathscr{F}_{\Gamma}$ :

$$
\left\|\mathscr{F}_{\Gamma}\right\|=\left\|\mathscr{F}_{\Gamma}(F)\right\|_{\infty}=\mathscr{F}_{\Gamma}(F)(1)
$$

since $\mathscr{F}(F)(1)=\sum F^{(j)}(0) / j !=\sum \hat{F}(j)$. We rewrite Berman's equation in the form

$$
\begin{aligned}
\mathscr{F}_{\Gamma} & =\frac{\alpha+\pi}{2 \pi}\left[\frac{1}{\alpha+\pi} \int_{-\pi}^{\alpha} T_{-s} P T_{s} d s\right]+\frac{\pi-\alpha}{2 \pi}\left[\frac{1}{\pi-\alpha} \int_{\alpha}^{\pi} T_{-s} P T_{s} d s\right] \\
& =\theta P_{1}+(1-\theta) P_{2},
\end{aligned}
$$

where $0<\theta<1$ and $P_{1}, P_{2}$ are minimal projections onto $\pi_{\Gamma}$. We now let $P$ stand for either $P_{1}$ or $P_{2}$. For simpler notation we let $\hat{x}$ be the linear functional defined by $\hat{x} f=f\left(e^{i x}\right),-\pi \leqslant x \leqslant \pi$. Hence we have

$$
\begin{aligned}
\left\|\mathscr{F}_{\Gamma}\right\| & =\hat{x} \mathscr{F}_{\Gamma} T_{-x}(F)=\theta \hat{x} P_{1} T_{-x}(F)+(1-\theta) \hat{x} P_{2} T_{-x}(F) \\
& \leqslant \theta\left\|P_{1}\right\|+(1-\theta)\left\|P_{2}\right\|=\left\|\mathscr{F}_{\Gamma}\right\| .
\end{aligned}
$$

Thus, $\hat{x} P T_{-x}(F)=\left\|\mathscr{F}_{\Gamma}\right\|$ for each $x$. Let $H\left(x, e^{i t}\right)$ be the best $H_{0}^{1}$ approximation to $\sum g_{j}(t) e^{i j x}$ for each $x$ in $[-\pi, \pi]$. Then

$$
\begin{aligned}
\|\hat{x} P\|= & \text { distance from } \sum e^{i j x} d \mu_{j}(t) \text { to } H_{0}^{1} \text { in space of measures } \\
= & L^{1} \text { distance from } \sum g_{j}(t) e^{i j x} \text { to } H_{0}^{1} \text { plus measure norm of } \sum e^{i j x} d \rho_{j}(t) \\
= & \hat{x} P T_{-x}(F) \\
= & \int F\left(e^{i(t-x)}\right)\left[\sum g_{j}(t) e^{i j x}+H\left(x, e^{i t}\right)\right] d t \\
& +\int F\left(e^{i(t-x)}\right)\left[\sum e^{i j x} d \rho_{j}(t)\right] \\
< & \left\|\sum g_{j}(t) e^{i j x}+H\left(x, e^{i t}\right)\right\|_{L^{1}(d t)}+\left\|\sum e^{i j x} d \rho_{j}(t)\right\| \\
= & \|\hat{x} P\| .
\end{aligned}
$$

Thus, we conclude that

$$
0 \leqslant F\left(e^{i(t-x)}\right)\left[\sum g_{j}(t) e^{i j x}+H\left(x, e^{i t}\right)\right] \text { a.e. } d t
$$


and

$$
F\left(e^{i(t-x)}\right) \sum e^{i j x} d \rho_{j}(t) \text { is a nonnegative measure. }
$$

Equations (1.6) and (1.7) hold for every $x \in[-\pi, \pi]$. We shall use equations (1.6) and (1.7) to show that $g_{j}(t)=e^{-i j t}$ and $\rho_{j}=0$ for all $j$. We make mention here of the fact that since $P$ is a projection

$$
\int_{T} e^{i k t} d \mu_{j}(t)=\delta_{j k} \quad \text { for } j, k \in \Gamma
$$

2. Analysis of the minimal kernel. Associated with the extremal problem (1.5) for bounded holomorphic functions is an extremal kernel $K$. In the case $\Gamma=$ $\{0, \ldots, n\}$ we have both of the following formulas for $K$ :

$$
\left\{\begin{array}{l}
\text { (a) } K\left(e^{i t}\right)=\sum_{j=0}^{n} e^{-i j t}+J\left(e^{i t}\right), \\
\text { (b) } K(z)=z^{-n} \prod_{j=1}^{n}\left(1-\alpha_{j} z\right)^{2}, \quad z \in \mathbf{C} .
\end{array}\right.
$$

Here $J$ is the best $H_{0}^{1}$ approximation to $\sum e^{-i j t}$ and $0<\left|\alpha_{j}\right|<1,1<j<n$ (the latter is shown in §3; see equation (11), p. 138 and Example (i), p. 139, of [4]). We also know from the basic theory of extremal problems (again see [4, Chapter 8]) that

$$
0 \leqslant F\left(e^{i t}\right) K\left(e^{i t}\right) \text { for all } t
$$

so that in our case

$$
0 \leqslant e^{-i n \theta} F\left(e^{i \theta}\right) \prod_{1}^{n}\left(1-\alpha_{j} e^{i \theta}\right)^{2} \quad \text { a.e. } d \theta .
$$

We now exploit equations (1.6) and (1.10) to reach the desired conclusions. We first note that

$$
0 \leqslant e^{i n t} e^{-i n x} G\left(e^{i t} e^{-i x}\right)\left[\sum g_{j}(t) e^{i j x}+H\left(x, e^{i t}\right)\right],
$$

where we have written

$$
G(z)=1 / \prod_{1}^{n}\left(1-\alpha_{j} z\right)^{2} .
$$

Multiply equation (1.11) by $e^{i r t}+e^{-i r t}$ for $0<r<n$ and then integrate on $t$. The result is a real-valued function of the variable $e^{i x}$. The integral

$$
\int_{0}^{2 \pi} e^{i n t}\left(e^{i r t}+e^{-i r t}\right) G\left(e^{i t} e^{-i x}\right) H\left(x, e^{i t}\right) d t
$$

vanishes identically in $x$ since $H$ lies in $H_{0}^{1}$, since $G$ is analytic for $|z|<1$, and since $0 \leqslant r \leqslant n$. Replacing $x$ by $-x$ in (1.11) we see that the function

$$
e^{i n x} \int_{0}^{2 \pi} e^{i n t}\left(e^{i r t}+e^{-i r t}\right) G\left(e^{i t} e^{i x}\right)\left[\sum g_{j}(t) e^{-i j x}\right] d t,
$$

which is real-valued, has only nonnegative powers of $e^{i x}$ and hence must be identically constant. The same holds if we multiply (1.11) by $e^{i r t}-e^{-i r t}$ for 
$0 \leqslant r \leqslant n$ (except the resulting function is purely imaginary). Hence, we find that

$$
w_{r}\left(e^{i x}\right)=\int_{0}^{2 \pi} e^{i n t} e^{i r t} G\left(e^{i t} e^{i x}\right)\left[\sum g_{j}(t) e^{i(n-j) x}\right] d t
$$

is identically constant for $r=0, \pm 1, \ldots, \pm n$. Now

$$
G\left(e^{i \theta}\right)=\sum_{\nu=0}^{\infty} C_{\nu} e^{i \nu \theta},
$$

where the series is absolutely convergent since $G(z)$ is holomorphic on $|z|<1$. Substituting this into the expression (1.13) for $w_{r}$ and remembering that $w_{r}$ is a constant, we have for $m=1,2, \ldots$ and $r=0, \pm 1, \ldots, \pm n$,

$$
0=\sum_{\nu+n-j=m} C_{\nu} \int_{T} g_{j}(t) e^{i n t} e^{i n t} e^{i v t} d t
$$

Set $C_{\nu}=0$ if $\nu<0$; then (1.14) may be rewritten as

$$
0=\int_{T}\left(\sum C_{m+j-n} g_{j}(t) e^{i j t}\right) e^{i m t} e^{i r t} d t, \quad m=1,2, \ldots, r=0, \pm 1, \ldots, \pm n .
$$

To make the notation even simpler, set $g_{j} \equiv 0$ if $j \notin \Gamma$; then

$$
0=\int_{T}\left(\sum_{j=0}^{2 n-1} C_{m-n+j} g_{j}(t) e^{i j t}\right) e^{i m t} e^{i r t} d t .
$$

Fix $M \geqslant n+1$; set $m=M+s$ and $r=-s$ for $s=0, \pm 1, \ldots, \pm n$. Thus

$$
0=\int_{T}\left(\sum_{j=0}^{2 n-1} C_{M-n+s+j} g_{j}(t) e^{i j t}\right) e^{i M t} d t, \quad s=0, \pm 1, \ldots, \pm n .
$$

We now refer to Proposition 2 in $\$ 3$ to conclude that the Hankel matrix $\left\|C_{p+i+k}\right\|_{i, k=0}^{2 n-1}, p=-n+1,-n+2, \ldots$, has a nonzero determinant. Thus, if $J \in \Gamma$ is fixed, there are scalars $A_{-n}, \ldots, A_{n-1}$ with

$$
\sum_{s=-n}^{n-1} A_{s} C_{M-n+s+j}= \begin{cases}1, & j=J \\ 0, & j \neq J\end{cases}
$$

Hence,

$$
0=\int_{T}\left(g_{J}(t) e^{i J t}\right) e^{i M t} d t, \quad J \in \Gamma
$$

for each $M \geqslant n+1$. This argument may be repeated with $\sum e^{i j x} d \rho_{j}(t)$ in place of $\sum e^{i j x} g_{j}(t)+H\left(x, e^{i t}\right)$; the conclusion reached is that

$$
0=\int_{T} e^{i J t} e^{i M t} d \rho_{J}(t), \quad M>n+1, J \in \Gamma .
$$

Hence, the measure $e^{i J t} e^{i n t} d \rho_{J}(t)$ is absolutely continuous with respect to Lebesgue measure by the F. and M. Riesz theorem [4, p. 41]. Since this measure is also singular with respect to Lebesgue measure it must vanish and hence $\rho_{J}=0$ for $J \in \Gamma$.

We now know that for each $j \in \Gamma, g_{j}(t)=R_{j}\left(e^{i t}\right)+h_{j}\left(e^{i t}\right)$ where $R_{j}$ is in the linear span of $\left\{e^{-i k t}: k=0, \ldots, 2 n\right\}$ and $h_{j}$ lies in $H_{0}^{1}$. Let us absorb the term 
$\sum h_{j}\left(e^{i t}\right) e^{i j x}$ into $H\left(x, e^{i t}\right)$. We then know that

$$
0 \leqslant \frac{\sum R_{j}\left(e^{i t}\right) e^{i j x}+H\left(x, e^{i t}\right)}{e^{i t} e^{-i x} K\left(e^{i t} e^{-i x}\right)} \quad \text { a.e. } d t \text { for each } x,
$$

where $K$ is given in (1.9). Let $x$ be held fixed. Multiply top and bottom of (1.18) by $e^{2 i n t}$ and make use of (1.9) to obtain

$$
0 \leqslant \frac{S\left(e^{i t}\right)+H_{1}\left(e^{i t}\right)}{e^{i n t} Q\left(e^{i t}\right)} \quad \text { a.e. } d t,
$$

where

$$
\begin{aligned}
S\left(e^{i t}\right) & =\sum_{j} e^{2 i n t} R_{j}\left(e^{i t}\right) e^{i j x}, \\
H_{1}\left(e^{i t}\right) & =e^{2 i n t} H\left(x, e^{i t}\right), \\
Q\left(e^{i t}\right) & =e^{i n x} \prod_{1}^{n}\left(1-\alpha_{j} e^{i t} e^{-i x}\right)^{2} .
\end{aligned}
$$

Now each of $S$ and $Q$ is a polynomial in $e^{i t}$ of degree $2 n$ or less and $H_{1}$ is in $H_{0}^{1}$ so that the Schwarz reflection principle immediately implies that $\left(S(z)+H_{1}(z)\right) / z^{n} Q(z)$ is a rational function of $z$ and thus the same holds for $H_{1}(z)$. Consequently, $S+H_{1}$ is rational on the sphere and holomorphic on $|z|<1$ while $Q$ is a polynomial of degree $2 n$ with zeros of order 2 at the points $e^{i x} / \alpha_{1}, \ldots, e^{i x} / \alpha_{n}, 0<\left|\alpha_{j}\right|<1$. Consider the rational function

$$
z^{n} \frac{\bar{S}(1 / \bar{z})+\bar{H}_{1}(1 / \bar{z})}{\bar{Q}(1 / \bar{z})}
$$

where the bar denotes complex conjugation. When $|z|=1$ this rational function equals the complex conjugate of $\left(S(z)+H_{1}(z)\right) / z^{n} Q(z)$. But the latter is realvalued by $(1.18)^{\prime}$. Hence these two rational functions coincide. Thus,

$$
z^{2 n}\left[\bar{S}(1 / \bar{z})+\bar{H}_{1}(1 / \bar{z})\right] /\left[S(z)+H_{1}(z)\right]=\bar{Q}(1 / \bar{z}) / Q(z)
$$

for all $z$. The right-hand side of (1.19) has a pole of order two at $e^{i x} / \alpha_{j}$ for $1 \leqslant j \leqslant n$. Since $0<\left|\alpha_{j}\right|<1$, we see that $S+H_{1}$ must have a zero of order two at $e^{i x} / \alpha_{j}$. Hence, $S+H_{1}=M Q$, where $M$ is rational, holomorphic on $|z|<1$ (and depends on $x$ ). Thus, from (1.19) we obtain

$$
M(z)=z^{2 n} \bar{M}(1 / \bar{z})
$$

so that $M$ is a polynomial of degree at most $2 n$ of the form

$$
M(z)=M(z, x)=c_{n}(x) z^{n}+\sum_{k=0}^{n-1}\left\{c_{k}(x) z^{k}+\bar{c}_{k}(x) z^{2 n-k}\right\}
$$

where $c_{n}$ is real. We shall show that $c_{n}=1$ and $c_{k}=0$ for $k=0, \ldots, n-1$.

Returning to the definitions of $S, H_{1}$, and $Q$ we find that

$$
e^{i n t}\left\{\sum_{j=0}^{n} R_{j}\left(e^{i t}\right) e^{i j x}+H\left(x, e^{i t}\right)\right\}=e^{-i n t} e^{i n x} M\left(e^{i t}, x\right) \prod_{1}^{n}\left(1-\alpha_{j} e^{i t} e^{-i x}\right)^{2} .
$$


Let $r$ be an integer, $0 \leqslant r \leqslant n$, and refer to (1.13). We then compute

$$
\begin{aligned}
w_{r}\left(e^{i x}\right) & =\int_{0}^{2 \pi} e^{i n t} e^{i r t} G\left(e^{i t} e^{i x}\right)\left\{\sum_{j=0}^{n} R_{j}\left(e^{i t}\right) e^{i(n-j) x}\right\} d t \\
& =\int_{0}^{2 \pi} e^{i r t} e^{-i n t} M\left(e^{i t},-x\right) d t \\
& =c_{n-r}(-x), \quad 0 \leqslant r \leqslant n .
\end{aligned}
$$

But $w_{r}$ is constant. Thus, $c_{k}$ is constant for $k=0, \ldots, n$. Now

$$
\begin{aligned}
w_{r} & =\frac{1}{2 \pi} \int_{0}^{2 \pi} w_{r}\left(e^{i x}\right) d x=\int_{0}^{2 \pi} e^{i n t} e^{i r t} R_{n}\left(e^{i t}\right) G(0) d t \\
& = \begin{cases}0, & 0<r \leqslant n, \\
1, & r=0,\end{cases}
\end{aligned}
$$

by (1.8). Thus, $M\left(e^{i t}, x\right)=e^{i n t}$. Next refer to (1.21) and conclude that

$$
\sum_{j=0}^{n} R_{j}\left(e^{i t}\right) e^{i j x}+H\left(x, e^{i t}\right)=e^{-i n t} e^{i n x} \prod_{j=1}^{n}\left(1-\alpha_{j} e^{i t} e^{-i x}\right)^{2} .
$$

However, the right-hand side of (1.22) is precisely $K\left(e^{i t} e^{-i x}\right)$ by (1.9)(b). Thus, using (1.9)(a) we have

$$
\sum_{j=0}^{n} R_{j}\left(e^{i t}\right) e^{i j x}+H\left(x, e^{i t}\right)=\sum_{j=0}^{n} e^{-i j t} e^{i j x}+J\left(e^{i t} e^{-i x}\right) .
$$

Since both $H\left(x, e^{i t}\right)$ and $J\left(e^{i t} e^{-i x}\right)$ lie in $H_{0}^{1}$ for each $x$ as functions of $e^{i t}$, we see that

$$
\sum_{j=0}^{n} R_{j}\left(e^{i t}\right) e^{i j x}=\sum_{j=0}^{n} e^{-i j t} e^{i j x} \text { for all } t, x .
$$

Finally, a comparison of Fourier coefficients of $e^{i j x}$ yields $R_{j}\left(e^{i t}\right)=e^{-i j t}$, the desired conclusion.

\section{Details.}

Proposition 1. Let $\left(\sum_{k=0}^{n} z^{k}\right)^{1 / 2}=\sum_{0}^{\infty} \lambda_{j} z^{j}$ for $|z|$ small. Then $0<\lambda_{n}<\cdots<$ $\lambda_{0}$.

Proof. $\sum_{0}^{n} z^{k}=\left(1-z^{n+1}\right)(1-z)^{-1}$ so that $\left(\sum_{0}^{n} z^{k}\right)^{1 / 2}=(1-z)^{-1 / 2}+o\left(z^{n}\right)$ for $|z|$ small. However, $(1-z)^{-1 / 2}=\sum_{j=0}^{\infty} A_{j} z^{j}$ where

$$
\begin{aligned}
& A_{\nu}=\frac{1}{\nu !} \frac{(2 \nu-1) \ldots(3)(1)}{2^{\nu}}, \quad \nu=1,2,3 \ldots, \\
& A_{0}=1
\end{aligned}
$$

and $\lambda_{\nu}=A_{\nu}$ for $0 \leqslant \nu \leqslant n$.

Corollary. If $\Gamma=\{0,1,2, \ldots, n\}$, then $K$ has the form (1.9) and hence $\mathscr{F}_{\Gamma}$ is the unique projection onto $\pi_{\Gamma}$ of minimal norm.

Proof. According to the discussion on p. 140 of [4] it is sufficient to show that if

$$
\left(\sum_{j \in \Gamma} z^{n-j}\right)^{1 / 2}=\sum_{0}^{\infty} \lambda_{j} z^{j} \quad \text { for }|z| \text { small }
$$


then $P_{n}(z)=\lambda_{0}+\lambda_{1} z+\cdots+\lambda_{n} z^{n}$ does not vanish in $|z|<1$. However, $\Sigma_{\Gamma} z^{n-j}$ $=\Sigma_{\Gamma} z^{j}$ and so $P_{n}(z)=\sum_{k=0}^{n} \lambda_{k} z^{k}$, where $\lambda_{0}, \ldots, \lambda_{n}$ are determined by

$$
\left(\sum_{k=0}^{n} z^{k}\right)^{1 / 2}=\lambda_{0}+\lambda_{1} z+\cdots+\lambda_{n} z^{n}+\ldots
$$

According to Proposition $1,1=\lambda_{0}>\cdots>\lambda_{n}>0$ and so $P_{n}$ has no zeros in $|z| \leqslant 1$ by the well-known condition of Eneström-Kakeya (see [10, p. 136]).

Proposition 2. Let $0<\left|a_{k}\right|<1$ for $k=1, \ldots$, n and set

$$
G(z)=\prod_{1}^{n}\left(1-a_{k} z\right)^{-2}=\sum_{\nu=0}^{\infty} C_{\nu} z^{\nu}, \quad|z| \leqslant 1 .
$$

Let $C_{\nu}=0$ if $\nu<0$. Then for $p=-2 n+1,-2 n+2, \ldots$ the $(2 n) \times(2 n)$ matrix $\left\|C_{p+j+k}\right\|_{j, k=0}^{2 n-1}$ has rank $2 n$.

Proof. Replace $z$ by $1 / z$ in the expansion of $G$; then

$$
\frac{z^{2 n-1}}{\Pi_{1}^{n}\left(z-a_{k}\right)^{2}}=\sum_{0}^{\infty} C_{\nu} / z^{\nu+1}, . \quad|z|>1 .
$$

If $p=0,1,2, \ldots$, then

$$
\sum_{\nu=0}^{\infty} \frac{C_{p+\nu}}{z^{\nu+1}}=z^{p} \frac{z^{2 n-1}}{\Pi_{1}^{n}\left(z-a_{k}\right)^{2}}-\sum_{j=0}^{p-1} C_{j} z^{p-1-j} .
$$

The right-hand side of (1.23) is a rational function with precisely $2 n$ poles. If $p=-1,-2, \ldots,-2 n+1$, then we have

$$
\frac{C_{0}}{z^{1-p}}+\frac{C_{1}}{z^{2-p}}+\ldots=\frac{z^{2 n-1+p}}{\Pi_{1}^{n}\left(z-\alpha_{k}\right)^{2}} .
$$

The right-hand side of $(1.24)$ is also a rational function with precisely $2 n$ poles. In both cases, then, we may conclude from [5, Theorem 8, p. 207] that the infinite Hankel matrix $\left\|C_{p+j+k}\right\|_{j, k \geqslant 0}$ has rank $2 n$ and then from [5, Corollary, p. 206] that

$$
\operatorname{det}\left\|C_{p+j+k}\right\|_{j, k=0}^{2 n-1} \neq 0 \text {. }
$$

(We wish to thank Professor R. G. Douglas for suggesting [5] as a source of information on the rank of Hankel matrices.)

EXAmple 1. NonUNiQueness. The uniqueness of $\mathscr{F}_{\Gamma}$ as a projection of minimal norm can fail.

Let $\Gamma$ be the multiples of $r>1: \Gamma=\{0, r, 2 r, \ldots, m r\}$. Let $H_{0}$ be the best $H_{0}^{1}$ approximation to $D\left(e^{i \theta}\right)=\sum_{k=0}^{m} e^{-i r k \theta}$; note $H_{0}$ is a function of $r \theta$. Let $0<l<r$ and set

$$
(\tilde{P} f)\left(e^{i x}\right)=\int_{\Upsilon}(1+\cos l \theta)\left[D(\theta-x)+H_{0}(\theta-x)\right] f\left(e^{i \theta}\right) d \theta .
$$

Since $(\cos l \theta) e^{i r \theta} f\left(e^{i \theta}\right)$ lies in $H^{\infty}$, it follows that $P$ has range in $\pi_{\Gamma}$. It also follows that $\tilde{P}$ is a projection not equal to $\mathscr{F}_{\Gamma}$. Finally,

$$
\|\tilde{P}\| \leqslant \max _{x} \int|1+\cos l \theta|\left|D(\theta-x)+H_{0}(\theta-x)\right| d \theta=\left\|\mathscr{F}_{\Gamma}\right\|
$$

since $1+\cos l \theta \geqslant 0$ and $\cos l \theta$ convolved with a function of $r \theta$ is identically zero. 


\section{Part II. Minimal PRojections: COFinite CASE}

4. The major result. Let $\Gamma$ be a finite set of integers symmetric about 0 and let $\Lambda=Z \backslash \Gamma$. Define $\pi_{\Gamma}$ and $\pi_{\Lambda}$ to be the closed real linear span of $\{\cos j t, \sin j t$ : $j \in \Gamma\}$ and $\{\cos j t, \sin j t: j \in \Lambda\}$, respectively. In this part of the paper we provide a characterization of these (symmetric) sets $\Lambda$ for which the Fourier projection $\mathscr{F}_{\Lambda}$ is the unique minimal norm projection of $C(T)$ onto $\pi_{\Lambda}$. In this part all functions considered are real-valued.

Definition. An element $g \in \pi_{\Gamma}$ is determined by its zeros if, whenever $h$ is an element of $\pi_{\Gamma}$ and $h / g$ is continuous on $T$, then $h$ is a constant multiple of $g$. That is, if $\lambda_{1}, \ldots, \lambda_{m}$ is a listing of the zeros of $g$, counting multiplicities, then the only elements of $\pi_{\Gamma}$ which vanish at $\lambda_{1}, \ldots, \lambda_{m}$ are the constant multiples of $g$. We remark also that $g$ being determined by its zeros is equivalent to: whenever $h$ is in $\pi_{\Gamma}$ with $\operatorname{sgn}(g(s)+\delta h(s))=\operatorname{sgn} g(s)$ a.e. $-d s$, for $|\delta| \leqslant 1$, then $h$ is a constant multiple of $g$.

Set

$$
D_{\Gamma}(t)=\sum_{j \in \Gamma} e^{i j t} .
$$

The major result of this part is the following.

THEOREM. Let $\Gamma$ be a finite set of integers, symmetric about 0 . The Fourier projection $\mathscr{F}_{\Lambda}$ is the unique minimal norm projection of $C(T)$ onto $\pi_{\Lambda}$ if and only if $D_{\Gamma}$ is determined by its zeros.

Proof. Let us assume first that $D_{\Gamma}$ is not determined by its zeros. We will show that it is possible to construct a projection $P$ onto $\pi_{\Lambda}$ with $\|P\|=\left\|\mathscr{F}_{\Lambda}\right\|$ but yet $P \neq \mathscr{F}_{\Lambda}$. The assumption that $D_{\Gamma}$ is not determined by its zeros implies that the subspace of $\pi_{\Gamma}$ consisting of functions which vanish at the zeros of $D_{\Gamma}$, including multiplicities, is at least two dimensional. Hence, there is a function $h \in \pi_{\Gamma}$ with $h / D_{\Gamma}$ continuous on $T$ and

$$
\int_{T} h \operatorname{sgn} D_{\Gamma}=0
$$

Consequently,

$$
\operatorname{sgn}\left(D_{\Gamma}+\varepsilon h\right)=\operatorname{sgn} D_{\Gamma}
$$

if $|\varepsilon|<\left\|h / D_{\Gamma}\right\|_{\infty}^{-1}$. Thus, for the same range of $\varepsilon$,

$$
\int\left|D_{\Gamma}+\varepsilon h\right|=\int\left(D_{\Gamma}+\varepsilon h\right) \operatorname{sgn}\left(D_{\Gamma}+\varepsilon h\right)=\int\left|D_{\Gamma}\right|
$$

by (2.2) and (2.3). Hence, $\left\|D_{\Gamma}+\varepsilon h\right\|_{1}=\left\|\mathscr{F}_{\Gamma}\right\|$ for all small $\varepsilon$. Now choose a nonzero function $\varphi \in C(T)$ with

$$
\int \varphi(s) e^{i(n-m) s} d s=0, \quad \text { for all } n, m \in \Gamma .
$$

There is no loss in assuming $\|\varphi\|_{\infty}<\left\|h / D_{\Gamma}\right\|_{\infty}^{-1}$. It follows from (2.5) that

$$
\int \varphi(s) e^{-i k s} h(s-t) d s=0
$$


for all $t$ and all $k \in \Gamma$. Now define an operator $P$ by

$$
\begin{aligned}
(P f)(s) & =f(s)-\int\left[D_{\Gamma}(s-t)+\varphi(s) h(s-t)\right] f(t) d t \\
& =\left(\mathscr{F}_{\Lambda} f\right)(s)-\varphi(s) \int h(s-t) f(t) d t .
\end{aligned}
$$

We must show that $P$ is a projection onto $\pi_{\Lambda}$ and $\|P\|=\left\|\mathscr{F}_{\Lambda}\right\|$. First suppose $k \in \Gamma$; then

$$
\begin{aligned}
(P f)^{\wedge}(k) & =\int(P f)(s) e^{-i k s} d s \\
& =-\int f(t) \int \varphi(s) h(s-t) e^{-i k s} d s d t \\
& =0
\end{aligned}
$$

by (2.6). Thus, the range of $P$ lies in $\pi_{\Lambda}$. Next, let $f=e^{i m s}$ for some $m \in \Lambda$. Then

$$
P f=\mathscr{F}_{\Lambda} f-\varphi(s) \int h(s-t) e^{i m t} d t=f
$$

since $h \in \pi_{\Gamma}$. Consequently, $P$ is a projection onto $\pi_{\Lambda}$. Finally, we compute the norm of $P$ :

$$
\begin{aligned}
\|P\| & \leqslant 1+\sup _{s \in[0,2 \pi]} \int\left|D_{\Gamma}(s-t)+\varphi(s) h(s-t)\right| d t \\
& =1+\left\|\mathscr{F}_{\Gamma}\right\|=\left\|\mathscr{F}_{\Lambda}\right\| .
\end{aligned}
$$

The first equality results from (2.4) and the second from the observation that if $S$ is any finite rank operator on a space $C(X)$, with $X$ perfect, then

$$
\|I+S\|=1+\|S\| \text {. }
$$

This, in turn, follows readily from the fact that $S$ has a representation $S f=$ $\sum \varphi_{i}(f) f_{i}$, for some $\varphi_{i}$ 's in $C(X)^{*}, f_{i}$ 's in $C(X)$. We shall have further use of (2.7) in what follows. This concludes the proof that if $\mathscr{F}_{\Lambda}$ is the unique minimal norm projection onto $\pi_{\Lambda}$ then $D_{\Gamma}$ is determined by its zeros.

Now for the proof that if $D_{\Gamma}$ is determined by its zeros then $\mathscr{F}_{\Lambda}$ is unique. Using Berman's formula (see the Introduction for an explanation of the validity of Berman's formula in this setting) as in $\S 1$ we obtain that if $\mathscr{F}$ is not uniquely minimal then it is not an extreme point of the convex set of projections of least norm. We show that this contradicts the assumption that $D_{\Gamma}$ is determined by its zeros.

Since $\mathscr{F}_{\Lambda}$ is not extreme there exists a nonzero operator $Q: C_{2 \pi} \rightarrow C_{2 \pi}$ such that $P_{\delta}=F_{\Lambda}-\delta Q$ is a projection of least norm onto $\pi_{\Lambda}$ for all $\delta$ in $[-1,1]$. For each such $\delta$, define

$$
L_{\delta}=I-P_{\delta}=\mathscr{F}_{\Gamma}+\delta Q .
$$

Now observe that $Q$ is an operator with range in $\pi_{\Lambda}$ such that

(i) $Q$ annihilates $\pi_{\Lambda}$;

(ii) $Q$ has a kernel representation constructed as follows. For $j \in \Gamma$, let $h_{j}=$ $Q\left(e^{i j x}\right)$. By (i), if $f \in C_{2 \pi}$, 


$$
\begin{aligned}
Q f(t) & =Q\left(\mathscr{F}_{\Gamma} f\right)(t)=Q\left(\sum_{\Gamma} \hat{f}(j) e^{i j x}\right)(t) \\
& =\int f(s) \sum_{\Gamma} e^{-i j s} h_{j}(t) d s .
\end{aligned}
$$

Then $H(s, t)=\Sigma_{\Gamma} e^{-i j s} h_{j}(t)$ is the desired kernel. It follows now that if we define $K_{\delta}(s, t)=D_{\Gamma}(t-s)+\delta H(s, t),|\delta| \leqslant 1$, we have $L_{\delta} f(t)=\int f(s) K_{\delta}(s, t) d s$. Now $L_{\delta}$ has finite rank so, by (2.7), $\left\|L_{\delta}\right\|=\left\|P_{\delta}\right\|-1=\left\|\mathscr{F}_{\Lambda}\right\|-1=\left\|\mathscr{F}_{\Gamma}\right\|$. Also we obviously have $\left\|L_{\delta}\right\|=\sup _{t} \int\left|K_{\delta}(s, t)\right| d s$. Now define $\sigma(s)=\operatorname{sgn} D_{\Gamma}(s)$. Then we have

$$
\left\|\mathscr{F}_{\Gamma}\right\|=\int\left|D_{\Gamma}(t-s)\right| d s=\int \sigma(t-s) D_{\Gamma}(t-s) d s,
$$

for all $t$. Note that

$$
\int H(s, t) \sigma(t-s) d s=0, \text { for all } t .
$$

It follows that, for all $t$,

$$
\left\|\mathscr{F}_{\Gamma}\right\| \geqslant \int\left|K_{\delta}(s, t)\right| d s \geqslant \int K_{\delta}(s, t) \sigma(t-s) d s=\left\|\mathscr{F}_{\Gamma}\right\|,
$$

so that

$$
\left\|\mathscr{F}_{\Gamma}\right\|=\int\left|K_{\delta}(s, t)\right| d s=\int \sigma(t-s) K_{\delta}(s, t) d s .
$$

Hence, for all $t, \operatorname{sgn} K_{\delta}(s, t)=\sigma(t-s)$ a.e. $-d s$. By the remark made following the definition of "determined by its zeros", it follows that, for each $t$, there is a constant $c_{t}$ such that

$$
H(s, t)=c_{t} D_{\Gamma}(t-s), \quad \text { for all } s
$$

But then

$$
\begin{aligned}
\left|1+\delta c_{t}\right| \int\left|D_{\Gamma}(t-s)\right| d s & =\int\left|K_{\delta}(s, t)\right| d s \\
& =\int\left|D_{\Gamma}(t-s)\right| d s,
\end{aligned}
$$

implying that $c_{t}=0$ for all $t$. This means that $Q=0$, which is a contradiction. The proof is now complete.

It is interesting that the condition on $D_{\Gamma}$ of being determined by its zeros has been shown to be sufficient for the unique minimality of the Fourier projection from $L^{1}[-\pi, \pi]$ onto $\pi_{\Gamma}[7]$.

COROllary. Let $m$ be the number of zeros of $D_{\Gamma}$, counting multiplicities. If $m<\operatorname{dim} \pi_{\Gamma}$, then $\mathscr{F}_{\Lambda}$ is not the unique minimal norm projection onto $\pi_{\Lambda}$.

\section{Some examples.}

ExAmple 2. NonuniQueness. Let $\Gamma=\{0, \pm 2, \pm 3, \pm 4\}$; we will show that $D_{\Gamma}$ is not determined by its zeros by showing that $D_{\Gamma}$ has only 4 zeros, counting multiplicities. Since $\pi_{\Gamma}$ is 7 dimensional there will be many elements of $\pi_{\Gamma}$ which have the same zeros as $D_{\Gamma}$, including multiplicities, but which are not constant 
multiples of $D_{\Gamma}$. Now with $x=\cos t$

$$
D_{\Gamma}(t)=1+2 T_{2}(x)+2 T_{3}(x)+2 T_{4}(x)=p(x),
$$

where $T_{j}$ is the $j$ th Chebyshev polynomial of the first kind. Hence,

$$
\frac{1}{2} p(x)=8 x^{4}+4 x^{3}-6 x^{2}-3 x+\frac{1}{2} \text {. }
$$

Some computation shows that $p$ has only 2 roots in $[-1,1]$ and that $p^{\prime}$ is not zero at these roots. Thus, $D_{\Gamma}$ has just 4 zeros in $[0,2 \pi)$.

EXAmple 3. Uniqueness. Here are some sets $\Gamma$ for which $D_{\Gamma}$ is determined by its zeros.

(a) $\Gamma=\{0, \pm 1, \ldots, \pm n\}$; here $D_{\Gamma}(t)=D_{n}(t)=\sin ((n+1 / 2) t) / \sin (t / 2)$ has $2 n$ distinct zeros. Since no nonzero element of $\pi_{\Gamma}$ can have more than this many zeros, $D_{\Gamma}$ is determined by its zeros.

(b) Fix an integer $r \geqslant 2$ and let $\Gamma=\{0, \pm r, \ldots, \pm m r\}$ then $D_{\Gamma}(x)=D_{m}(r x)$. If $h \in \pi_{\Gamma}$, then $h(x)=Q(r x)$ for some $Q \in \pi_{\Gamma^{\prime}}$ where

$$
\Gamma^{\prime}=\{0, \pm 1, \ldots, \pm m\} \text {. }
$$

Hence, if $h / D_{\Gamma}$ is continuous then so is $Q / D_{m}$ and so $Q=\lambda D_{m}, \lambda=$ scalar, by (a). Thus, $h=\lambda D_{\Gamma}$, and $D_{\Gamma}$ is determined by its zeros.

(c) $\Gamma=\{-m, m\}$; here $D_{\Gamma}(x)=2 \cos m x$ and so $D_{\Gamma}$ has $2 m$ distinct zeros which is the most that any element of $\pi_{\Gamma}$ can have.

We wish to thank the referees for their helpful comments.

\section{REFERENCES}

1. D. L. Berman, On the impossibility of constructing a linear polynomial operator furnishing an approximation of the order of best approximation, Dokl. Akad. Nauk SSSR 120 (1958), 143-148.

2. W. Cheney, Introduction to approximation theory, McGraw-Hill, New York, 1966.

3. W. Cheney, C. R. Hobby, P. D. Morris, F. Schurer and D. E. Wulbert, On the minimal property of the Fourier projection, Trans. Amer. Math. Soc. 143 (1969), 249-258.

4. P. Duren, Theory of $H^{p}$ spaces, Academic Press, New York, 1970.

5. F. R. Gantmacher, The theory of matrices, Vol. II, Chelsea, New York, 1959.

6. P. V. Lambert, Minimum norm property of the Fourier projection in spaces of continuous functions, Bull. Soc. Math. Belg. 21 (1969), 359-369.

7. Minimum norm property of the Fourier projection in $L^{1}$-spaces, Bull. Soc. Math. Belg. 21 (1969), 370-391.

8. S. M. Lozinski, On a class of linear operators, Dokl. Akad. Nauk SSSR 61 (1948), 193-196.

9. J. Marcinkiewicz, Quelques remarques sur l'interpolation, Acta Litt. Sci. (Szeged) 8 (1937), 127-130.

10. M. Marden, The geometry of polynomials, Math. Surveys, no. 3, Amer. Math. Soc., Providence, R. I., 1966.

Department of Mathematics, Northwestern University, Evanston, Iluinois 60201

Department of Mathematics, Pennsylvania State University, University Park, Pennsylvania 16802

Department of Mathematics, University of California, San Diego, la Jolla, California 92093 